\title{
Predictors of Successful Weaning from Noninvasive Ventilation in Patients with Acute Exacerbation of Chronic Obstructive Pulmonary Disease: A Single-Center Retrospective Cohort Study
}

\author{
Jie Yu ${ }^{1,2} \cdot$ Meng-Rui Lee ${ }^{3} \cdot$ Chung-Ting Chen ${ }^{4,5} \cdot$ Yi-Tsung Lin ${ }^{1,6} \cdot$ Chorng-Kuang How ${ }^{1,4,5,7}$ (I)
}

Received: 26 April 2021 / Accepted: 15 August 2021 / Published online: 21 August 2021

(c) The Author(s), under exclusive licence to Springer Science+Business Media, LLC, part of Springer Nature 2021

\begin{abstract}
Purpose Noninvasive ventilation (NIV) is often required for patients with acute exacerbation of chronic obstructive pulmonary disease (AECOPD), and it can significantly reduce the need for endotracheal intubation. Currently, there is no standard method for predicting successful weaning from NIV. Therefore, we aimed to evaluate whether a weaning index can predict NIV outcomes of patients with AECOPD.

Methods This study was conducted at a single academic public hospital in northern Taiwan from February 2019 to January 2021. Patients with AECOPD admitted to the hospital with respiratory failure who were treated with NIV were included in the study. Univariate and multivariate logistic regression analyses were used to identify independent predictors of successful weaning from NIV. Receiver operating characteristic curve methodology was used to assess the predictive capacity. Results A total of 85 patients were enrolled, $65.9 \%$ of whom were successfully weaned from NIV. The patients had a mean age of 75.8 years and were mostly men (89.4\%). The rapid shallow breathing index (RSBI) $(P<0.001)$, maximum inspiratory pressure $(P=0.014)$, and maximum expiratory pressure $(P=0.004)$ of the successful group were significant while preparing to wean. The area under the receiver operating characteristic curve for the RSBI was 0.804 , which was considered excellent discrimination.

Conclusion The RSBI predicted successful weaning from NIV in patients with AECOPD with hypercapnic respiratory failure. This index may be useful for selecting patients with AECOPD that are suitable for NIV weaning.
\end{abstract}

Keywords Chronic obstructive pulmonary disease $\cdot$ Noninvasive ventilation $\cdot$ Weaning $\cdot$ Weaning index

Chorng-Kuang How

ckhow@vghtpe.gov.tw

1 Institute of Emergency and Critical Care Medicine, National Yang Ming Chiao Tung University, Taipei, Taiwan, ROC

2 National Taiwan University Hospital Hsin-Chu Branch, Hsinchu City, Taiwan, ROC

3 Department of Internal Medicine, National Taiwan University Hospital Hsin-Chu Branch, Hsinchu City, Taiwan, ROC

4 School of Medicine, National Yang Ming Chiao Tung University, Taipei, Taiwan, ROC

5 Emergency Department, Taipei Veterans General Hospital, \#201, Sec. 2, Shipai Rd., Beitou Dist., Taipei 11217, Taiwan, ROC

6 Division of Infectious Diseases, Department of Medicine, Taipei Veterans General Hospital, Taipei, Taiwan, ROC

7 Kinmen Hospital, Ministry of Health and Welfare, Kinmen, Taiwan, ROC

\section{Introduction}

Acute exacerbation of chronic obstructive pulmonary disease (AECOPD), a common cause of respiratory failure during hospital admission and readmission, is a public health problem that is associated with increased medical costs. AECOPD is characterized by persistent airflow limitation and develops when significant exposure to irritants causes an inflammatory response in the lungs [1]. The World Health Organization reported that COPD was the fifth most common disease globally in 2020 [2], with a prevalence of $7.8-19.7 \%$ in adults [3]. Approximately, $60 \%$ of patients with COPD admitted with hypercapnic respiratory failure die in hospital $[4,5]$.

The 1990s saw a wealth of research emerge on the characteristics of spontaneous breathing trials and how they relate to trial performance. Studies showed that noninvasive ventilation (NIV) was successful as first-line treatment for 
patients admitted to hospital with hypercapnic respiratory failure due to AECOPD [6-9]. COPD treated with NIV has lower inpatient mortality rates, shorter lengths of stay, and lower costs than treatment with invasive mechanical ventilation (IMV) [9, 10].

The weaning index for IMV is the most appropriate method to determine readiness for weaning training. However, no standard method has been shown to be a good predictor of weaning success with NIV, and there is no consensus among researchers regarding the extent and nature of weaning from NIV. It is possible that delayed weaning may expose the patient to unnecessary discomfort, increased risk of complications, and increased cost of care. The rapid shallow breathing index (RSBI) was introduced by Yang and Tobin in 1991. They found that there was a higher probability of weaning success if the RSBI was $\leq 105$ and spontaneous breathing trials were successful [11,12]. Once NIV therapy is initiated, however, the timing of withdrawal must be considered. Thus, we conducted a prospective observational study to investigate the use of a weaning index to predict weaning outcomes for patients with AECOPD requiring NIV.

\section{Materials and Methods}

\section{Study Design and Population}

To identify predictors of successful weaning from NIV for patients with AECOPD, we conducted a prospective, noninterventional study at the National Taiwan University Hospital Hsin-Chu Branch, a 637-bed academic public hospital in Hsinchu City, Taiwan. Consecutive patients (aged $\geq 20$ years) hospitalized with AECOPD between February 2019 and January 2021 who were treated with NIV during hospitalization using a bi-level positive airway pressure mode (VPAP III ST-A; ResMed, UK) and a full facemask were enrolled. NIV was initiated based on the patient's respiratory status and indications, including respiratory acidosis (partial pressure of $\mathrm{CO}_{2} \geq 45 \mathrm{mmHg}$; arterial $\mathrm{pH} \leq 7.35$ ), severe dyspnea with clinical signs of respiratory muscle fatigue, or persistent hypoxemia despite supplemental oxygen therapy [13]. Subsequent ICU admission for identified cases depended on current ICU availability and the clinician's discretionary judgement, and medical treatment was provided by a respiratory therapist. The exclusion criteria were cardiovascular instability, lack of patient cooperation or a Glasgow Coma Scale (GCS) score $<13$, increased aspiration risk, recent facial trauma, upper airway obstruction, increased sputum secretion, or respiratory arrest. Patients who required NIV post-extubation, were pregnant, were transferred from another acute care facility, required long-term continuous NIV, or signed do-not-resuscitate orders were also excluded [14-16].

\section{Weaning Criteria and Protocol}

After admission, NIV was administered $24 \mathrm{~h}$ /day, except during meals and for expectoration. The initial inspiratory (IPAP) and expiratory (EPAP) positive airway pressures were determined based on achieving acceptable arterial blood gas $(\mathrm{ABG})$ parameters, a respiratory rate $(\mathrm{RR})<25$ breaths/minute, and patient tolerance and comfort. All patients had an initial IPAP set at $12 \mathrm{cmH}_{2} \mathrm{O}$, which was gradually increased by $2-3 \mathrm{cmH}_{2} \mathrm{O}$, as tolerated, but did not exceed $25 \mathrm{cmH}_{2} \mathrm{O}$. The EPAP was initially set at $5 \mathrm{cmH}_{2} \mathrm{O}$ and then gradually increased by $1-2 \mathrm{cmH}_{2} \mathrm{O}$, as needed, to improve hypoxemia [17]. Decisions regarding the duration of NIV and whether to progress to endotracheal intubation were made by the clinical team, based on the attending physician's judgement.

The criteria and weaning protocols by Duan and Momii were modified for use in this study $[18,19]$. The weaning criteria used were adequate mentation, oxygen saturation $\left(\mathrm{SpO}_{2}\right) \geq 90 \%$ on a fraction of inspired oxygen $\left(\mathrm{FiO}_{2}\right) \leq 0.4$, $\mathrm{pH} \geq 7.30$, a systolic blood pressure of $90-180 \mathrm{mmHg}$ without vasopressor support, a body temperature of $36-38{ }^{\circ} \mathrm{C}$, and a heart rate of 50-120 bpm [18]. Patients who met these criteria were enrolled in the weaning protocol.

The weaning protocol was performed by decreasing the IPAP and EPAP by $3 \mathrm{cmH}_{2} \mathrm{O}$ every $30 \mathrm{~min}$, with close monitoring for worsening $\mathrm{SpO}_{2}$ and/or RR. When the IPAP and EPAP were reduced to 15 and $5 \mathrm{cmH}_{2} \mathrm{O}$, respectively, with a satisfactory $\mathrm{SpO}_{2}(\geq 90 \%)$ on a $\mathrm{FiO}_{2} \leq 0.4$, NIV was withdrawn and $3 \mathrm{~L} / \mathrm{min}$ oxygen was administered via a nasal cannula for an additional $30 \mathrm{~min}$ [19]. The primary outcome was successful weaning from NIV. Weaning failure was determined by objective or subjective determination of respiratory failure based on the need for repeated NIV, intubation, high-flow nasal cannula oxygen therapy, or longterm NIV support. Weaning failure was also determined in the case of patient death within $48 \mathrm{~h}$ of NIV weaning [20].

\section{Data Collection and Outcomes}

Patient demographic data, including past medical history, physiological measurements, blood test results (including data on baseline arterial blood gases), COPD-associated therapy, hospital areas, other therapies, initial setting of NIV, and respiratory indices [maximal inspiratory (MIP) and expiratory (MEP) pressure, tidal volume (TV), RR, and RSBI], were extracted from medical records. The RSBI was measured using a Wright respirometer ("nSpire" Wright/ Haloscale Respirometers; Hertford, UK). Patients were asked to breathe through the respirometer for $1 \mathrm{~min}, \mathrm{RR}$ and 
TV were measured, and RR was divided by TV to calculate the RSBI. MIP and MEP were measured with a respiratory pressure force meter ("MTC" Gas Pressure Gauge, Taoyuan, ROC). MIP was measured with a maximum inspiratory effort maintained for $\geq 1 \mathrm{~s}$, and MEP was measured with a maximum respiratory effort maintained for $\geq 1 \mathrm{~s}$. All parameters were recorded during the first $2 \mathrm{~h}$ of NIV treatment and before NIV was turned off within $2 \mathrm{~h}$. The primary outcome was successful weaning from NIV, which was determined by the number of patients no longer requiring NIV support during their hospital stay. The secondary outcome was the length of hospital stay (days).

\section{Statistical Analyses}

Categorical variables are expressed as $N(\%)$. Descriptive data are reported as mean \pm standard deviation or median [interquartile range (IQR)]. Variables were evaluated for an association with NIV weaning outcome using Pearson's chi-squared test (or Fisher's exact test, when appropriate) for categorical data and the Student's $t$ test for variables with normal distribution (or Mann-Whitney $U$ test as non-parametric methods) for numerical data. Univariate analysis was performed to determine predictive factors for successful weaning from NIV. Variables with $P<0.2$ were entered into the multivariable logistic regression analysis to identify independent predictors of NIV weaning outcome. Odds ratios (ORs) and 95\% confidence intervals (CIs) were reported for all independent predictors. Receiver operating characteristic (ROC) curve analysis was performed to determine the capacity to predict the success of NIV weaning for different variables. All statistical analyses were conducted using IBM SPSS software version 22.0 (IBM Co., Armonk, NY, USA). A two-tailed $P<0.05$ was considered statistically significant.

\section{Results}

During the study period, 141 eligible patients with AECOPD received NIV treatment upon admission; 56 were excluded due to their post-extubation status, non-cooperation or GCS score $(<13)$, episodes of respiratory arrest, or requirement for long-term continuous NIV support (Fig. 1). Eighty-five patients met the inclusion criteria, including $56(65.9 \%)$ in the NIV weaning success group and $29(34.1 \%)$ in the NIV weaning failure group. The average age of the patients was $75.8 \pm 10.1$ years; $76(89.4 \%)$ patients were men. Additional baseline characteristics are presented in Table 1. No significant differences were detected between the two groups in terms of sex, age, body mass index, GCS score, white blood cell count, COPD severity, Acute Physiology and Chronic Health Evaluation II score, hospital areas, C-reactive protein (CRP) level, albumin level, COPD-associated therapy, therapeutic interventions (systemic steroids and antibiotics), ABG data before the initiation of NIV, and initial setting of NIV. There were significant differences between the NIV weaning success and failure groups in terms of smoking history (50 vs. 20 cases; $P=0.020$ ), days on NIV until weaning ( 2.0 vs. 3.0 days; $P=0.019)$, average duration of NIV treatment (5.0 vs. 13.0 days; $P<0.001)$, and length of hospital stay (13.0 vs. 17.0 days; $P=0.008$ ).
Fig. 1 Flowchart of participant recruitment. AECOPD, acute exacerbation of chronic obstructive pulmonary disease; $G C S$ Glasgow Coma Scale; NIV noninvasive ventilation

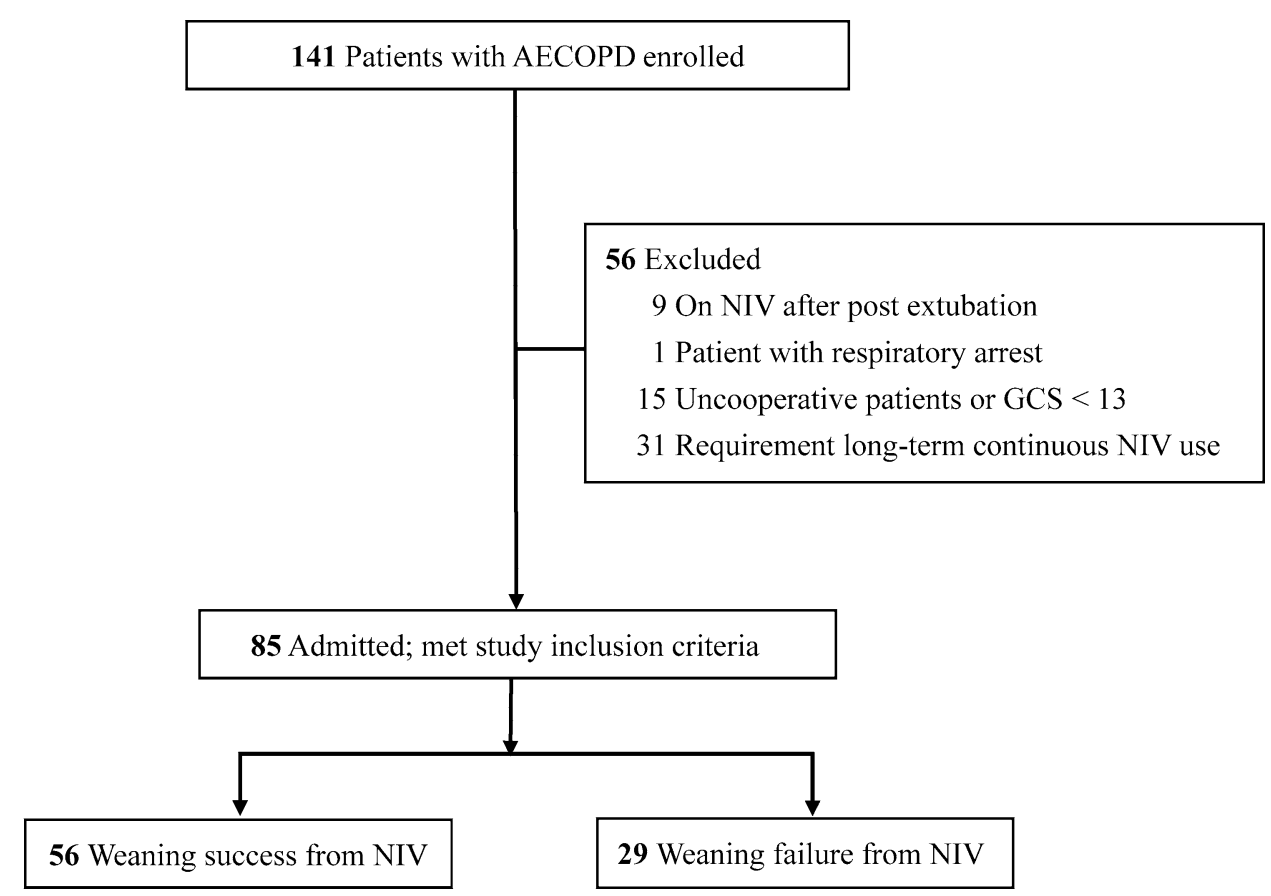


Table 1 Baseline characteristics of the study population

\begin{tabular}{|c|c|c|c|c|}
\hline \multirow[t]{2}{*}{ Characteristic } & \multirow[t]{2}{*}{ Total $(N=85)$} & \multicolumn{3}{|l|}{ NIV weaning } \\
\hline & & Success $(N=56)$ & Failure $(N=29)$ & \\
\hline Sex, male $(\%)^{\mathrm{b}}$ & $76(89.4)$ & $52(92.9)$ & $24(82.8)$ & 0.263 \\
\hline Age, mean (SD), years ${ }^{\mathrm{a}}$ & $75.8 \pm 10.1$ & $76.0 \pm 10.4$ & $75.4 \pm 9.8$ & 0.786 \\
\hline BMI, mean (SD), $\mathrm{kg} / \mathrm{m}^{2 \mathrm{a}}$ & $22.7 \pm 6.5$ & $22.4 \pm 5.8$ & $23.3 \pm 7.9$ & 0.595 \\
\hline GCS, median $(\mathrm{IQR})^{\mathrm{a}}$ & $15.0(15.0-15.0)$ & $15.0(15.0-15.0)$ & $15.0(15.0-15.0)$ & 0.722 \\
\hline Smoking history, $N(\%)^{\mathrm{b}}$ & $70(82.4)$ & $50(89.3)$ & $20(69.0)$ & $0.020 *$ \\
\hline WBC, median (IQR), K/ $\mu \mathrm{L}^{\mathrm{a}}$ & $9.4(7.1-13.5)$ & $9.4(7.4-13.9)$ & $9.6(6.7-12.5)$ & 0.704 \\
\hline $\mathrm{CRP}$, median (IQR), $\mathrm{mg} / \mathrm{dL}^{\mathrm{a}}$ & $3.5(1.2-9.6)$ & $3.0(0.7-9.5)$ & $5.2(1.5-10.5)$ & 0.219 \\
\hline Albumin, median (IQR), g/dL ${ }^{\mathrm{a}}$ & $3.2(3.0-3.5)$ & $3.3(3.1-3.5)$ & $3.2(2.7-3.4)$ & 0.089 \\
\hline COPD GOLD assessment (\%) & & & & 0.105 \\
\hline Group A and $\mathrm{B}, N(\%)^{\mathrm{b}}$ & $24(28.2)$ & $19(33.9)$ & $5(17.2)$ & \\
\hline Group C and D, $N(\%)^{\mathrm{b}}$ & $61(71.8)$ & $37(66.1)$ & $24(82.8)$ & \\
\hline APACHE II, median (IQR) ${ }^{\mathrm{a}}$ & $12.0(8.5-15.0)$ & $12.0(9.0-15.0)$ & $12.0(7.5-15.0)$ & 0.824 \\
\hline Days on NIV until weaning, median (IQR), days ${ }^{\mathrm{a}}$ & $2.0(2.0-4.0)$ & $2.0(2.0-3.0)$ & $3.0(2.0-5.0)$ & $0.019 *$ \\
\hline Average duration of NIV treatment, median (IQR), days ${ }^{\mathrm{a}}$ & $6.0(4.0-12.0)$ & $5.0(3.0-7.0)$ & $13.0(9.5-22.0)$ & $<0.001 *$ \\
\hline LOS, median (IQR), day ${ }^{\mathrm{a}}$ & $13.0(9.5-20.5)$ & $13.0(9.0-17.0)$ & $17.0(12.0-26.0)$ & $0.008^{*}$ \\
\hline \multicolumn{5}{|l|}{ Hospital area } \\
\hline Diagnosis area & & & & 0.120 \\
\hline Emergency room, $N(\%)^{\mathrm{b}}$ & $46(54.1)$ & $33(58.9)$ & $13(44.8)$ & \\
\hline General ward, $N(\%)^{\mathrm{b}}$ & $32(37.6)$ & $17(30.4)$ & $15(51.7)$ & \\
\hline Intensive care unit, $N(\%)^{\mathrm{b}}$ & $7(8.2)$ & $6(10.7)$ & $1(3.4)$ & \\
\hline Treatment area & & & & 0.547 \\
\hline Emergency room, $N(\%)^{\mathrm{b}}$ & $2(2.4)$ & $2(3.6)$ & $0(0.0)$ & \\
\hline General ward, $N(\%)^{\mathrm{b}}$ & $55(64.7)$ & $35(62.5)$ & $20(69.0)$ & \\
\hline Intensive care unit, $N(\%)^{\mathrm{b}}$ & $28(32.9)$ & $19(33.9)$ & $9(31.0)$ & \\
\hline \multicolumn{5}{|l|}{ Comorbidities } \\
\hline Diabetes mellitus, $N(\%)^{\mathrm{b}}$ & $39(45.9)$ & $29(51.8)$ & $10(34.5)$ & 0.129 \\
\hline Hypertension, $N(\%)^{\mathrm{b}}$ & $52(61.2)$ & $34(60.7)$ & $18(62.1)$ & 0.903 \\
\hline Congestive heart failure, $N(\%)^{\mathrm{b}}$ & $11(12.9)$ & $5(8.9)$ & $6(20.7)$ & 0.174 \\
\hline Coronary artery disease, $N(\%)^{\mathrm{b}}$ & $14(16.5)$ & $11(19.6)$ & $3(10.3)$ & 0.363 \\
\hline Arrhythmia, $N(\%)^{\mathrm{b}}$ & $16(18.8)$ & $9(16.1)$ & $7(24.1)$ & 0.367 \\
\hline Chronic renal disease, $N(\%)^{\mathrm{b}}$ & $11(12.9)$ & $8(14.3)$ & $3(10.3)$ & 0.742 \\
\hline Benign prostatic hyperplasia, $N(\%)^{\mathrm{b}}$ & $16(18.8)$ & $8(14.3)$ & $8(27.6)$ & 0.137 \\
\hline Malignancies other than lung cancer, $N(\%)^{\mathrm{b}}$ & $10(11.8)$ & $5(8.9)$ & $5(17.2)$ & 0.298 \\
\hline Lung cancer, $N(\%)^{\mathrm{b}}$ & $6(7.1)$ & $4(7.1)$ & $2(6.9)$ & $>0.999$ \\
\hline \multicolumn{5}{|l|}{ COPD therapy } \\
\hline Inhaled SABA, $N(\%)^{\mathrm{b}}$ & $41(48.2)$ & $28(50.0)$ & $13(44.8)$ & 0.651 \\
\hline Inhaled SAMA, $N(\%)^{\mathrm{b}}$ & $28(32.9)$ & $17(30.4)$ & $11(37.9)$ & 0.481 \\
\hline Inhaled LABA, $\mathrm{N}(\%)^{\mathrm{b}}$ & $54(63.5)$ & $34(60.7)$ & $20(69.0)$ & 0.454 \\
\hline Inhaled LAMA, $N(\%)^{\mathrm{b}}$ & $53(62.4)$ & $34(60.7)$ & $19(65.5)$ & 0.665 \\
\hline Inhaled corticosteroids, $N(\%)^{\mathrm{b}}$ & $36(42.4)$ & $25(44.6)$ & $11(37.9)$ & 0.553 \\
\hline Long-term oxygen therapy, $N(\%)^{\mathrm{b}}$ & $7(8.2)$ & $3(5.4)$ & $4(13.8)$ & 0.223 \\
\hline Pulmonary rehabilitation, $N(\%)^{\mathrm{b}}$ & $17(20.0)$ & $12(21.4)$ & $5(17.2)$ & 0.647 \\
\hline \multicolumn{5}{|l|}{ Therapeutic interventions } \\
\hline Systemic corticosteroids, $N(\%)^{\mathrm{b}}$ & $74(87.1)$ & $50(89.3)$ & $24(82.8)$ & 0.499 \\
\hline Antibiotics, $N(\%)^{\mathrm{b}}$ & $74(87.1)$ & $51(91.1)$ & $23(79.3)$ & 0.174 \\
\hline \multicolumn{5}{|l|}{ NIV initial setting } \\
\hline IPAP, median (IQR), $\mathrm{cmH}_{2} \mathrm{O}^{\mathrm{a}}$ & $16.0(15.0-18.5)$ & $16.0(15.0-19.7)$ & $16.0(15.0-18.0)$ & 0.783 \\
\hline EPAP, median (IQR), $\mathrm{cmH}_{2} \mathrm{O}^{\mathrm{a}}$ & $5.0(5.0-6.0)$ & $5.0(5.0-6.0)$ & $5.0(5.0-6.0)$ & 0.258 \\
\hline
\end{tabular}


Table 1 (continued)

\begin{tabular}{|c|c|c|c|c|}
\hline \multirow[t]{2}{*}{ Characteristic } & \multirow[t]{2}{*}{ Total $(N=85)$} & \multicolumn{3}{|l|}{ NIV weaning } \\
\hline & & Success $(N=56)$ & Failure $(N=29)$ & \\
\hline $\mathrm{FiO}_{2}$, median (IQR), $(\%)^{\mathrm{a}}$ & $35.0(34.0-44.5)$ & $35.0(33.0-40.0)$ & $35.0(35.0-50.0)$ & 0.094 \\
\hline \multicolumn{5}{|c|}{ Blood gas analysis (before the initiation of NIV) } \\
\hline Arterial $\mathrm{pH}$, mean $(\mathrm{SD})^{\mathrm{a}}$ & $7.31 \pm 0.07$ & $7.30 \pm 0.75$ & $7.32 \pm 0.88$ & 0.486 \\
\hline $\mathrm{PaO}_{2}$, median (IQR), $\mathrm{mmHg}^{\mathrm{a}}$ & $77.0(62.8-98.0)$ & $78.4(61.7-100.5)$ & $74.1(65.1-87.4)$ & 0.499 \\
\hline $\mathrm{PaCO}_{2}$, mean $(\mathrm{SD}), \mathrm{mmHg}^{\mathrm{a}}$ & $60.3 \pm 18.9$ & $60.7 \pm 18.7$ & $59.3 \pm 19.5$ & 0.743 \\
\hline $\mathrm{HCO}_{3}$, mean $(\mathrm{SD}), \mathrm{mEq} / \mathrm{L}^{\mathrm{a}}$ & $29.1 \pm 6.8$ & $29.3 \pm 6.7$ & $28.9 \pm 7.0$ & 0.801 \\
\hline $\mathrm{BE}, \operatorname{median}(\mathrm{IQR}), \mathrm{mmol} / \mathrm{L}^{\mathrm{a}}$ & $2.6(-0.7-7.0)$ & $2.5(-0.6-6.1)$ & $3.2(-2.2-9.7)$ & 0.677 \\
\hline $\mathrm{SaO}_{2}$, median (IQR), $\%^{\mathrm{a}}$ & $93(90-96)$ & $93.5(90.0-96.7)$ & $92(89.5-95.5)$ & 0.316 \\
\hline
\end{tabular}

APACHE II acute physiology and chronic health evaluation; $B M I$ body mass index; $B E$ bicarbonate; $C O P D$ chronic obstructive pulmonary disease; $C R P C$-reactive protein; $E P A P$ expiratory positive airway pressure; $\mathrm{FiO}_{2}$ fraction of inspired oxygen; $\mathrm{GCS}$ Glasgow Coma Scale; $\mathrm{HCO} 3$ bicarbonate; IPAP inspiratory positive airway pressure; IQR interquartile range; $L A B A$ long acting beta agonist; LAMA long acting muscarinic antagonist; $L O S$ length of hospital stay; $M E P$ maximal expiratory pressure; $M I P$ maximal inspiratory pressure; $N I V$ noninvasive ventilation; $\mathrm{PaO}_{2}$ partial pressure of oxygen; $\mathrm{PaCO}_{2}$ partial pressure of carbon dioxide; $S A B A$ short acting beta agonist; SAMA short acting muscarinic antagonist; $\mathrm{SaO}_{2}$ arterial oxygen saturation; $\mathrm{SD}$ standard deviation; $W B C$ white blood cell

${ }^{\mathrm{a}}$ Continuous variables are expressed as mean $\pm \mathrm{SD}$ or median (IQR)

${ }^{\mathrm{b}}$ Categorical variables are expressed as $N(\%)$

$* P<0.05$, significant

\section{Predictive Factors for Successful Weaning from NIV}

The weaning parameters and ABG findings of the two groups are shown in Table 2. Within $2 \mathrm{~h}$ of initiating NIV treatment, no significant differences were detected between the two groups in any of the weaning indices or ABG data. However, immediately before NIV was turned off within $2 \mathrm{~h}$, the NIV weaning success group was compared to the NIV weaning failure group, the NIV weaning success group had a lower RSBI, RR, and higher VE, TV, MIP, predict MIP, and MEP, predict MEP $(P<0.001,<0.001$, $0.016,<0.001,0.014,0.013$ and $0.004,0.012$, respectively). There was a significant difference between the two groups in the hospital mortality rate ( 3 vs. 9 cases; $P=0.002$ ), and there were no significant differences in readmission within 60 days of discharge.

\section{Univariate Analysis}

For the primary outcome of successful weaning from NIV, univariate analysis showed that higher MIP and MEP were associated with an increased rate of successful weaning [OR 1.026 (95\% CI 1.001-1.052); $P=0.040$ and OR 1.025 (95\% CI 1.003-1.048); $P=0.026$, respectively] after turning off NIV. Conversely, days on NIV until weaning and RSBI were inversely associated with successful weaning [OR 0.716 (95\% CI 0.539-0.949); $P=0.020$ and OR 0.970 (95\% CI 0.956-0.984); $P<0.001$, respectively] after turning off NIV.

\section{Multivariate Analysis}

We used a multivariable logistic model to examine selected factors, including sex, smoking history, albumin level, antibiotic use, COPD severity, MEP, RSBI, and days on NIV until weaning. MIP and MEP are highly correlated, as both are related to respiratory muscle strength. To address the issue of collinearity, we excluded MIP because it was less significant than MEP. Patients with a lower RSBI were significantly more likely to be weaned successfully [OR 0.976 (95\% CI 0.959-0.993); $P=0.006$ ] (Table 3). Hosmer and Lemeshow goodness-of-fit chi-squared test was 4.065 $[P=0.772(>0.05)]$.

\section{Predictive Value of the Weaning Index}

The ROC curve was analyzed by selecting the follow-up RSBI. The area under the ROC curve for NIV weaning was 0.804 (95\% CI 0.706-0.901) (Fig. 2). The RSBI at a threshold of 67.4 predicted successful weaning from NIV, with a sensitivity of $82.1 \%$, a specificity of $69.0 \%$, a positive likelihood ratio of 2.648, and a negative likelihood ratio of 0.377 (Table 4).

\section{Discussion}

NIV is now the mainstay of therapy before endotracheal intubation and mechanical ventilation for patients with acute respiratory distress or failure due to AECOPD [22]. 
Table 2 Weaning parameters and arterial blood gas findings between the two groups

\begin{tabular}{|c|c|c|c|c|}
\hline \multirow[t]{2}{*}{ Clinical outcome } & \multirow[t]{2}{*}{ Total $(N=85)$} & \multicolumn{2}{|l|}{ NIV weaning } & \multirow[t]{2}{*}{$P$ value } \\
\hline & & Success $(N=56)$ & Failure $(N=29)$ & \\
\hline \multicolumn{5}{|l|}{ Weaning index (on NIV within $2 \mathrm{~h}$ ) } \\
\hline RSBI, mean (SD), breaths/(min $\mathrm{mL})^{\mathrm{a}}$ & $103.1 \pm 60.0$ & $100.0 \pm 60.4$ & $109.1 \pm 59.7$ & 0.512 \\
\hline VE, median (IQR), $\mathrm{L}^{\mathrm{a}}$ & $6.3(4.8-9.1)$ & $7.0(5.1-9.1)$ & $5.1(4.2-9.3)$ & 0.100 \\
\hline $\mathrm{TV}$, median (IQR), $\mathrm{mL}^{\mathrm{a}}$ & $271.0(198.0-378.5)$ & $275.5(209.2-407.0)$ & $242.0(173.0-339.0)$ & 0.218 \\
\hline $\mathrm{RR}$, mean (SD), breaths $/ \mathrm{min}^{\mathrm{a}}$ & $24.8 \pm 6.9$ & $24.7 \pm 6.6$ & $25.0 \pm 7.5$ & 0.877 \\
\hline MIP, median (IQR), $-\mathrm{cmH}_{2} \mathrm{O}^{\mathrm{a}}$ & $30.0(20.0-40.0)$ & $34.0(20.0-40.0)$ & $26.0(17.0-38.0)$ & 0.205 \\
\hline $\mathrm{MEP}$, median (IQR), $+\mathrm{cmH}_{2} \mathrm{O}^{\mathrm{a}}$ & $36.0(22.0-44.0)$ & $36.0(22.5-48.0)$ & $36.0(21.5-41.5)$ & 0.263 \\
\hline \multicolumn{5}{|l|}{ Blood gas analysis (on NIV within $2 \mathrm{~h}$ ) } \\
\hline Arterial $\mathrm{pH}$, mean $(\mathrm{SD})^{\mathrm{a}}$ & $7.31 \pm 0.07$ & $7.31 \pm 0.06$ & $7.31 \pm 0.08$ & 0.707 \\
\hline $\mathrm{PaO}_{2}$, median (IQR), $\mathrm{mmHg}^{\mathrm{a}}$ & $90.0(78.5-113.1)$ & $91.1(79.4-114.1)$ & $88.0(75.5-114.0)$ & 0.777 \\
\hline $\mathrm{PaCO}_{2}$, median (IQR), $\mathrm{mmHg}^{\mathrm{a}}$ & $60.5(48.6-67.3)$ & $60.4(48.2-66.1)$ & $61.1(48.8-68.4)$ & 0.838 \\
\hline $\mathrm{HCO}_{3}$, mean $(\mathrm{SD}), \mathrm{mEq} / \mathrm{L}^{\mathrm{a}}$ & $29.3 \pm 6.9$ & $29.5 \pm 6.9$ & $28.9 \pm 7.0$ & 0.705 \\
\hline $\mathrm{BE}$, mean $(\mathrm{SD}), \mathrm{mmol} / \mathrm{L}^{\mathrm{a}}$ & $4.2 \pm 7.7$ & $4.0 \pm 7.0$ & $4.6 \pm 8.9$ & 0.733 \\
\hline $\mathrm{SaO}_{2}$, median (IQR), $(\%)^{\mathrm{a}}$ & $96.0(93.0-97.0)$ & $96.0(94.0-97.0)$ & $94.0(92.5-98.5)$ & 0.373 \\
\hline \multicolumn{5}{|l|}{ Weaning index (before turning off NIV within $2 \mathrm{~h}$ ) } \\
\hline RSBI, median (IQR), breaths/(min mL) $)^{\mathrm{a}}$ & $53.5(29.8-83.2)$ & $38.5(18.7-58.9)$ & $84.9(55.3-128.8)$ & $<0.001 *$ \\
\hline VE, median (IQR), $\mathrm{L}^{\mathrm{a}}$ & $7.4(5.9-9.9)$ & $7.9(6.4-10.2)$ & $6.2(5.5-8.1)$ & $0.016^{*}$ \\
\hline $\mathrm{TV}$, median (IQR), $\mathrm{mL}^{\mathrm{a}}$ & $408.0(272.5-571.0)$ & $444.5(360.2-671.2)$ & $264.0(219.0-395.5)$ & $<0.001 *$ \\
\hline $\mathrm{RR}$, mean (SD), breaths $/ \mathrm{min}^{\mathrm{a}}$ & $19.8 \pm 6.8$ & $17.7 \pm 6.2$ & $23.7 \pm 6.5$ & $<0.001 *$ \\
\hline MIP, median (IQR), $-\mathrm{cmH}_{2} \mathrm{O}^{\mathrm{a}}$ & $36.0(24.0-52.0)$ & $44.0(30.5-55.2)$ & $30.0(20.0-40.0)$ & $0.014 *$ \\
\hline Predict MIP, median (IQR), $(\%)^{\mathrm{a}, \mathrm{c}}$ & $42.0(29.0-61.0)$ & $49.0(33.2-67.7)$ & $35.0(24.0-47.0)$ & $0.013^{*}$ \\
\hline MEP, median (IQR), $+\mathrm{cmH}_{2} \mathrm{O}^{\mathrm{a}}$ & $44(28.0-60.0)$ & $48.5(40.0-67.0)$ & $28.0(20.0-50.0)$ & $0.004 *$ \\
\hline Predict MEP, median (IQR), $(\%)^{\mathrm{a}, \mathrm{c}}$ & $41.0(27.0-59.0)$ & $44.0(33.2-60.5)$ & $30.0(21.0-54.0)$ & $0.012 *$ \\
\hline \multicolumn{5}{|l|}{ Blood gas analysis (before turning off NIV within $2 \mathrm{~h}$ ) } \\
\hline Arterial $\mathrm{pH}$, mean $(\mathrm{SD})^{\mathrm{a}}$ & $7.41 \pm 0.49$ & $7.41 \pm 0.50$ & $7.42 \pm 0.48$ & 0.186 \\
\hline $\mathrm{PaO}_{2}$, median (IQR), $\mathrm{mmHg}^{\mathrm{a}}$ & $95.4 \pm 34.2$ & $92.3 \pm 30.5$ & $101.4 \pm 40.6$ & 0.257 \\
\hline $\mathrm{PaCO}_{2}$, mean $(\mathrm{SD}), \mathrm{mmHg}^{\mathrm{a}}$ & $45.7 \pm 10.8$ & $45.4 \pm 10.4$ & $46.4 \pm 11.6$ & 0.704 \\
\hline $\mathrm{HCO}_{3}$, median (IQR), mEq/L $\mathrm{L}^{\mathrm{a}}$ & $29.6(22.8-33.6)$ & $29.5(22.8-33.0)$ & $29.8(22.6-34.1)$ & 0.765 \\
\hline $\mathrm{BE}$, mean $(\mathrm{SD}), \mathrm{mmol} / \mathrm{L}^{\mathrm{a}}$ & $4.6 \pm 6.5$ & $4.0 \pm 6.3$ & $5.9 \pm 6.9$ & 0.235 \\
\hline $\mathrm{SaO}_{2}$, median (IQR), $(\%)^{\mathrm{a}}$ & $96.0(94.0-97.7)$ & $96.0(93.2-97.0)$ & $97.0(94.2-98.0)$ & 0.226 \\
\hline \multicolumn{5}{|l|}{ Clinical outcome (after NIV treatment) } \\
\hline In-hospital mortality, $N(\%)^{\mathrm{b}}$ & $12(14.1)$ & $3(5.4)$ & $9(31.0)$ & $0.002 *$ \\
\hline Readmission after discharge within 60 days, $N(\%)^{\mathrm{b}}$ & $28(32.9)$ & $20(35.7)$ & $8(27.6)$ & 0.450 \\
\hline
\end{tabular}

$B E$ bicarbonate; $I Q R$ interquartile range; $M E P$ maximal expiratory pressure; $M I P$ maximal inspiratory pressure; $N I V$ noninvasive ventilation; $\mathrm{PaO}_{2}$ partial pressure of oxygen; $\mathrm{PaCO}_{2}$ partial pressure of carbon dioxide; $R R$ respiratory rate; $R S B I$ rapid shallow breathing index; $T V$ tidal volume; $V E$ minute ventilation; $\mathrm{SaO}_{2}$ arterial oxyhemoglobin saturation; $S D$ standard deviation

${ }^{\mathrm{a}}$ Continuous variables are expressed as mean $\pm \mathrm{SD}$ or median $(\mathrm{IQR})$

${ }^{\mathrm{b}}$ Categorical variables are expressed as $N(\%)$

${ }^{\mathrm{c}}$ Including data from studies in the review by Evans et al. [21]. Male MIP reference: $120-(0.41 \times$ age $)$; female MIP reference: $108-(0.61 \times$ age $)$; male MEP reference: $174-(0.83 \times$ age $)$; female MEP reference: $131-(0.86 \times$ age $)$, percentage of predict MIP or MEP of percentage $=$ real data (MIP or MEP)/reference (MIP or MEP)

$* P<0.05$, significant

NIV is now recommended as a direct alternative for the management of patients with acute respiratory failure, particularly during the course of AECOPD, to preclude tracheal intubation and subsequent complications [6, 23, 24]. This is derived from the fact that, in many cases, patient outcomes (both short- and long-term) are better with NIV than with IMV $[25,26]$. There is strong evidence to suggest that this benefit is due to a reduction in the rate of ventilatorassociated pneumonia and avoidance of intensive care unit admission [27, 28]. Thus far, however, there is uncertainty 
Table 3 Univariate and multivariate analyses of clinical factors associated with successful weaning

\begin{tabular}{|c|c|c|c|c|}
\hline \multirow[t]{2}{*}{ Variable } & \multicolumn{2}{|l|}{ Univariate analysis } & \multicolumn{2}{|l|}{ Multivariate analysis } \\
\hline & OR (95\% CI) & $P$ value & OR (95\% CI) & $P$ value \\
\hline Age & $1.006(0.963-1.052)$ & 0.783 & - & - \\
\hline Sex (reference, female) & $2.708(0.667-10.993)$ & 0.163 & $1.274(0.154-10.527)$ & 0.822 \\
\hline Smoking history (reference, yes) & $0.267(0.084-0.847)$ & $0.025^{*}$ & $0.544(0.087-3.396)$ & 0.515 \\
\hline Days on NIV until weaning, days & $0.716(0.539-0.949)$ & $0.020^{*}$ & $0.825(0.562-1.211)$ & 0.326 \\
\hline Albumin, g/dL & $2.561(0.869-7.550)$ & 0.088 & $2.196(0.555-8.694)$ & 0.262 \\
\hline Systemic corticosteroids (reference, yes) & $1.736(0.481-6.261)$ & 0.399 & - & - \\
\hline Antibiotics (reference, yes) & $0.376(0.104-1.358)$ & 0.135 & $0.405(0.082-1.999)$ & 0.267 \\
\hline COPD group (reference, $\mathrm{C}+\mathrm{D}$ group) & $2.465(0.811-7.487)$ & 0.112 & $1.556(0.420-5.769)$ & 0.508 \\
\hline Off NIV RSBI, breaths/(min•mL) & $0.970(0.956-0.984)$ & $<0.001^{*}$ & $0.976(0.959-0.993)$ & $0.006^{*}$ \\
\hline Off NIV MIP, $-\mathrm{cmH}_{2} \mathrm{O}$ & $1.026(1.001-1.052)$ & $0.040^{*}$ & - & - \\
\hline Off NIV MEP; $+\mathrm{cmH}_{2} \mathrm{O}$ & $1.025(1.003-1.048)$ & $0.026^{*}$ & $0.996(0.968-1.023)$ & 0.755 \\
\hline
\end{tabular}

Obtained through enter model construction, including only significant independent predictors; OR of NIV treatment success associated with each variable in the model

$C I$ confidence interval; $C O P D$ chronic obstructive pulmonary disease; $M E P$ maximal expiratory pressure; $M I P$ maximal inspiratory pressure; $N I V$ noninvasive ventilation; $O R$ odds ratio; $R S B I$ rapid shallow breathing index

$* P<0.05$, significant

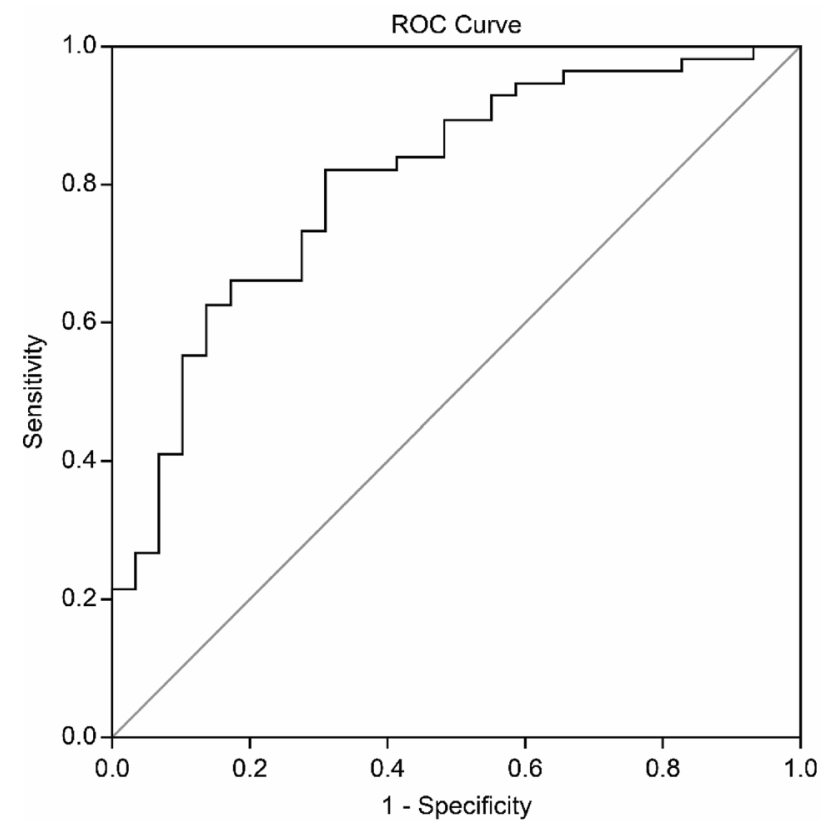

Fig. 2 Receiver operating characteristic (ROC) curve for weaning parameters. The sensitivity and specificity of the weaning parameter for each image set a priori operation point and are indicated on the curve. They are as follows: off RSBI images: sensitivity, $69.0 \%$; specificity, $82.1 \%$; area under the ROC curve, 0.804

regarding the best method of evaluating weaning from NIV, which generally requires the cooperation of the patient during the AECOPD recovery phase. Delayed weaning may potentially expose the patient to unnecessary discomfort and increase the risk of complications [29].
Although the RSBI was not a significant predictor of successful NIV [30], our results suggest that it is a reasonably good predictor of success for weaning a patient from not only IMV [31] but also NIV. The RSBI has been found to be an accurate index [32] that is calculated by dividing RR by TV in liters. Clinicians have successfully used this ratio in most mechanical ventilation weaning protocols. In a study by Berg et al. [15], patients were divided into two groups according to their RSBI. The assisted RSBI (aRSBI) is a criterion for weaning from IMV. The authors recorded it based on the initial level of support: high $(\mathrm{aRSBI} \geq 105)$ or low (aRSBI < 105). The RSBI may be useful for titrating the NIV settings, as an aRSBI $\geq 105$ is associated with the need for intubation and increased in-hospital mortality. According to Sellarer et al. [33], NIV can be discontinued immediately, without the need for a weaning period, allowing rapid discontinuation of NIV, if the patient's condition improves. However, our results suggest that a weaning period is essential for this to succeed.

In our study, using ROC curve analysis, an RSBI $\leq 67.4$ was associated with the highest sensitivity and specificity for determining successful weaning from NIV. In cases where chronic hypercapnia persists during the night for patients with AECOPD, NIV can be beneficial because it can provide gas exchange and improve night-time hypoventilation. It is not clear, however, whether long-term NIV can be used for stable chronic COPD patients with chronic comorbidities or pneumonia [34-36]. Patients with moderate-to-severe COPD frequently have persistent low-grade systemic inflammation with elevated levels of circulating inflammatory cascade molecules, such as CRP [37, 38]. Furthermore, some authors 
Table 4 Analysis of NIV treatment

\begin{tabular}{llllll}
\hline & AUC $(95 \% \mathrm{CI})$ & Optimal cutoff & Sensitivity $(\%)$ & Specificity $(\%)$ & $P$ at difference \\
\hline Off NIV RSBI, breaths/(min $\mathrm{mL})$ & $0.804(0.706-0.901)$ & 67.4 & 69.0 & 82.1 & $<0.001 *$ \\
\hline
\end{tabular}

Data are presented as AUC $(95 \% \mathrm{CI}) ; * P<0.05$, predicted outcome of a significant difference in weaning success

$A U C$ area under the receiver operating characteristic curve; $C I$ confidence interval; NIV noninvasive ventilation; RSBI rapid shallow breathing index

$* P<0.05$, significant

have reported that high CRP levels in patients with COPD are associated with worse short- and long-term clinical outcomes [39, 40]. In our study, we found that CRP levels were not significantly associated with weaning outcomes, indicating that the mechanism of successful weaning from NIV is very complex. This suggests that NIV weaning outcomes are not entirely mediated by modulation of the immune response, or determined by the effect of lung inflammation itself, in patients with COPD. Although CRP and B-type natriuretic peptide (BNP) have high diagnostic potency for COPD patients combined with cor-pulmonale, and are positively correlated with cardiac function classification [41], CRP level may not be useful in assessing weaning outcomes for COPD patients with NIV support [42].

Our study is limited by its small sample size and observational nature. Our hospital also does not have a distinct protocol outlining when to commence NIV. Dyspnea, one of the NIV initiation criteria, may also not be clearly defined. In the future, it may be possible to use a more objective assessment scale, such as the modified Medical Research Council dyspnea or Borg scale. In addition, cor-pulmonale and ejection fraction parameters were not evaluated in this cohort study, which may affect RSBI, MEP, and MIP when weaning from NIV. Both left ventricular and right ventricular functions may interact with intra-thoracic pressure in a complex and often unpredictable fashion. In future studies, these parameters may be included and collected for adjustment additional. Our patients may differ from those treated with NIV at other hospitals, even those with AECOPD. Finally, data were not collected after discharge. Therefore, an analysis of long-term outcomes was not possible.

\section{Conclusion}

We evaluated the use of potential factors and various respiratory indices for predicting successful weaning from NIV in patients with AECOPD with hypercapnic respiratory failure. A lower RSBI immediately before turning off NIV was more likely to be associated with successful weaning. Thus, RSBI is a single clinical parameter that can be easily measured at the bedside to help predict the likelihood of successful NIV weaning in patients with AECOPD. However, additional studies with longer follow-up periods and larger sample sizes should be conducted to further evaluate the predictive ability of the RSBI, or other potential weaning indices, for NIV in populations other than patients with COPD.

Acknowledgements We are grateful for the continued support of the Department of Thoracic Medicine, National Taiwan University Hospital Hsin-Chu Branch, and to National Yang Ming Chiao Tung University, Taiwan for editing the manuscript.

Author Contributions List the names across each section below: Category 1; Conception and design of study: JY, MRL, CKH, YTL. Acquisition of data: JY, MRL. Analysis and/or interpretation of data: JY, MRL, CKH. Category 2; Drafting of the manuscript: JY, CTC, CKH. Revising the manuscript critically for important intellectual content: JY, MRL, CTC, CKH.

Funding The authors did not receive support from any organization for the submitted work.

Data Availability Data are available from the Thoracic Medicine Unit of the National Taiwan University Hsin-Chu Hospital, Taiwan.

\section{Declarations}

Conflict of interest The authors have no relevant financial or non-financial interests to disclose.

Ethical Approval All procedures performed in studies involving human participants were in accordance with the ethical standards of the institutional and/or national research committee and with the 1964 Helsinki Declaration and its later amendments or comparable ethical standards. The study was approved by the hospital institutional review board.

Informed Consent Informed consent was obtained from all individual participants included in the study (or their next of kin).

Consent for Publication All participants have consented to the submission of the manuscript to the journal.

\section{References}

1. Qureshi H, Sharafkhaneh A, Hanania NA (2014) Chronic obstructive pulmonary disease exacerbations: latest evidence and clinical implications. Ther Adv Chronic Dis 5:212-227. https://doi.org/ 10.1177/2040622314532862

2. Ansari SF, Memon M, Brohi N, Tahir A (2019) Noninvasive positive pressure ventilation in patients with acute respiratory 
failure secondary to acute exacerbation of chronic obstructive pulmonary disease. Cureus 11:e5820. https://doi.org/10.7759/ CUREUS.5820

3. Zwerink M, Brusse-Keizer M, van der Valk PD, Zielhuis GA, Monninkhof EM, van der Palen J, Frith PA, Effing T (2014) Self management for patients with chronic obstructive pulmonary disease. Cochrane Database Syst Rev. https://doi.org/10.1002/14651 858.CD002990.PUB3

4. Lightowler JV, Wedzicha JA, Elliott MW, Ram FS (2003) Noninvasive positive pressure ventilation to treat respiratory failure resulting from exacerbations of chronic obstructive pulmonary disease: cochrane systematic review and meta-analysis. BMJ 326:185. https://doi.org/10.1136/BMJ.326.7382.185

5. Donaldson GC, Seemungal TA, Bhowmik A, Wedzicha JA (2002) Relationship between exacerbation frequency and lung function decline in chronic obstructive pulmonary disease. Thorax 57:847852. https://doi.org/10.1136/THORAX.57.10.847

6. Brochard L, Mancebo J, Wysocki M, Lofaso F, Conti G, Rauss A, Simonneau G, Benito S, Gasparetto A, Lemaire F, Isabey D (1995) Noninvasive ventilation for acute exacerbations of chronic obstructive pulmonary disease. N Engl J Med 333:817-822. https://doi.org/10.1056/NEJM199509283331301

7. Nicolini A, Ferrera L, Santo M, Ferrari-Bravo M, Del Forno M, Sclifò F (2014) Noninvasive ventilation for hypercapnic exacerbation of chronic obstructive pulmonary disease: factors related to noninvasive ventilation failure. Pol Arch Med Wewn 124:525531. https://doi.org/10.20452/PAMW.2460

8. Sanchez D, Smith G, Piper A, Rolls K (2014) Non-invasive ventilation guidelines for adult patients with acute respiratory failure: a clinical practice guideline (Version 1). Agency for Clinical Innovation NSW Government, Chatswood, NSW

9. Hill NS, Brennan J, Garpestad E, Nava S (2007) Noninvasive ventilation in acute respiratory failure. Crit Care Med 35:2402-2407. https://doi.org/10.1097/01.CCM.0000284587.36541.7F

10. Ambrosino N, Vagheggini G (2008) Noninvasive positive pressure ventilation in the acute care setting: where are we? Eur Respir J 31:874-886. https://doi.org/10.1183/09031936.00143507

11. Karthika M, Al Enezi FA, Pillai LV, Arabi YM (2016) Rapid shallow breathing index. Ann Thorac Med 11:167-176. https:// doi.org/10.4103/1817-1737.176876

12. McConville JF, Kress JP (2012) Weaning patients from the ventilator. N Engl J Med 367:2233-2239. https://doi.org/10.1056/ NEJMra1203367

13. Mirza S, Clay RD, Koslow MA, Scanlon PD (2018) COPD guidelines: a review of the 2018 GOLD report. Mayo Clin Proc 93:1488-1502. https://doi.org/10.1016/J.MAYOCP.2018.05.026

14. Soleimanpour H, Taghizadieh A, Salimi R, Golzari SE, Mahmoodpoor A, Safari S, Mehdizadeh Esfanjani RM, Heshmat Y (2014) Rapid shallow breathing index survey, a predictor of non-invasive ventilation necessity in patients with chronic obstructive pulmonary disease exacerbation: an analytical descriptive prospective study. Iran Red Crescent Med J 16:e13326. https://doi.org/10. 5812/IRCMJ.13326

15. Berg KM, Lang GR, Salciccioli JD, Bak E, Cocchi MN, Gautam S, Donnino MW (2012) The rapid shallow breathing index as a predictor of failure of noninvasive ventilation for patients with acute respiratory failure. Respir Care 57:1548-1554. https://doi. org/10.4187/RESPCARE.01597

16. Megahed MM, Habib TN, Dwidar E (2016) Rapid shallow breathing index as a predictor of ventilatory support necessity in patients with acute exacerbation of chronic obstructive pulmonary disease. Am J Res Commun 4:60-74. https://doi.org/10.11648/J.JA.20170 503.12

17. Carrillo A, Ferrer M, Gonzalez-Diaz G, Lopez-Martinez A, Llamas N, Alcazar M, Capilla L, Torres A (2012) Noninvasive ventilation in acute hypercapnic respiratory failure caused by obesity hypoventilation syndrome and chronic obstructive pulmonary disease. Am J Respir Crit Care Med 186:1279-1285. https://doi.org/ 10.1164/RCCM.201206-11010C

18. Duan J, Tang X, Huang S, Jia J, Guo S (2012) Protocol-directed versus physician-directed weaning from noninvasive ventilation: the impact in chronic obstructive pulmonary disease patients. J Trauma Acute Care Surg 72:1271-1275. https://doi.org/10.1097/ TA.0b013e318249a0d5

19. Momii H, Tashima Y, Kadokami T, Narita S, Yoshida M, Ando SI (2012) Experience of step-wise protocol using noninvasive positive pressure ventilation for treating cardiogenic pulmonary edema. Eur J Emerg Med 19:267-270. https://doi.org/10.1097/ MEJ.0b013e32834ada48

20. Boles JM, Bion J, Connors A, Herridge M, Marsh B, Melot C, Pearl R, Silverman H, Stanchina M, Vieillard-Baron A, Welte $\mathrm{T}$ (2007) Weaning from mechanical ventilation. Eur Respir J 29:1033-1056

21. Evans JA, Whitelaw WA (2009) The assessment of maximal respiratory mouth pressures in adults. Respir Care 54:1348-1359

22. Sinuff T, Keenan SP, Department of Medicine, McMaster University (2004) Clinical practice guideline for the use of noninvasive positive pressure ventilation in COPD patients with acute respiratory failure. J Crit Care 19:82-91. https://doi.org/10.1016/J.JCRC. 2004.04.006

23. Bott J, Carroll MP, Conway JH, Keilty SE, Ward EM, Brown AM, Paul EA, Elliott MW, Godfrey RC, Wedzicha JA, Moxham J (1993) Randomised controlled trial of nasal ventilation in acute ventilatory failure due to chronic obstructive airways disease. Lancet 341:1555-1557. https://doi.org/10.1016/0140-6736(93) 90696-e

24. Brochard L, Isabey D, Piquet J, Amaro P, Mancebo J, Messadi AA, Brun-Buisson C, Rauss A, Lemaire F, Harf A (1990) Reversal of acute exacerbations of chronic obstructive lung disease by inspiratory assistance with a face mask. N Engl J Med 323:15231530. https://doi.org/10.1056/NEJM199011293232204

25. Confalonieri M, Parigi P, Scartabellati A, Aiolfi S, Scorsetti S, Nava S, Gandola L (1996) Noninvasive mechanical ventilation improves the immediate and long-term outcome of COPD patients with acute respiratory failure. Eur Respir J 9:422-430. https://doi. org/10.1183/09031936.96.09030422

26. Martin TJ, Hovis JD, Costantino JP, Bierman MI, Donahoe MP, Rogers RM, Kreit JW, Sciurba FC, Stiller RA, Sanders MH (2000) A randomized, prospective evaluation of noninvasive ventilation for acute respiratory failure. Am J Respir Crit Care Med 161:807813. https://doi.org/10.1164/AJRCCM.161.3.9808143

27. Keenan SP, Gregor J, Sibbald WJ, Cook D, Gafni A (2000) Noninvasive positive pressure ventilation in the setting of severe, acute exacerbations of chronic obstructive pulmonary disease: more effective and less expensive. Crit Care Med 28:2094-2102. https://doi.org/10.1097/00003246-200006000-00072

28. Plant PK, Owen JL, Parrott S, Elliott MW (2003) Cost effectiveness of ward based non-invasive ventilation for acute exacerbations of chronic obstructive pulmonary disease: economic analysis of randomised controlled trial. BMJ 326:956. https://doi.org/10. 1136/BMJ.326.7396.956

29. Hall JB, Wood LD (1987) Liberation of the patient from mechanical ventilation. JAMA 257:1621-1628. https://doi.org/10.1001/ JAMA.257.12.1621

30. Lin MS, Guo HR, Huang MH, Chen CR, Wu CL (2008) Predictors of successful noninvasive ventilation treatment for patients suffering acute respiratory failure. J Chin Med Assoc 71:392-398. https://doi.org/10.1016/S1726-4901(08)70089-3

31. Krieger BP, Isber J, Breitenbucher A, Throop G, Ershowsky P (1997) Serial measurements of the rapid-shallow-breathing index as a predictor of weaning outcome in elderly medical patients. 
Chest 112:1029-1034. https://doi.org/10.1378/CHEST.112.4. 1029

32. Yang KL, Tobin MJ (1991) A prospective study of indexes predicting the outcome of trials of weaning from mechanical ventilation. N Engl J Med 324:1445-1450. https://doi.org/10.1056/ NEJM199105233242101

33. Sellares J, Ferrer M, Anton A, Loureiro H, Bencosme C, Alonso R, Martinez-Olondris P, Sayas J, Peñacoba P, Torres A (2017) Discontinuing noninvasive ventilation in severe chronic obstructive pulmonary disease exacerbations: a randomised controlled trial. Eur Respir J 50:28679605. https://doi.org/10.1183/13993 003.01448-2016

34. Lindenauer PK, Stefan MS, Shieh MS, Pekow PS, Rothberg MB, Hill NS (2014) Outcomes associated with invasive and noninvasive ventilation among patients hospitalized with exacerbations of chronic obstructive pulmonary disease. JAMA Intern Med 174:1982-1993. https://doi.org/10.1001/JAMAINTERNMED. 2014.5430

35. Mas A, Masip J (2014) Noninvasive ventilation in acute respiratory failure. Int J Chron Obstruct Pulmon Dis 9:837-852

36. Storre JH, Callegari J, Magnet FS, Schwarz SB, Duiverman ML, Wijkstra PJ, Windisch W (2018) Home noninvasive ventilatory support for patients with chronic obstructive pulmonary disease: patient selection and perspectives. Int J Chron Obstruct Pulmon Dis 13:753-760. https://doi.org/10.2147/COPD.S154718

37. Danesh J, Collins R, Appleby P, Peto R (1998) Association of fibrinogen, C-reactive protein, albumin, or leukocyte count with coronary heart disease: meta-analyses of prospective studies. JAMA 279:1477-1482. https://doi.org/10.1001/JAMA.279.18. 1477
38. Gan WQ, Man SF, Senthilselvan A, Sin DD (2004) Association between chronic obstructive pulmonary disease and systemic inflammation: a systematic review and a meta-analysis. Thorax 59:574-580. https://doi.org/10.1136/THX.2003.019588

39. Ruiz-González A, Lacasta D, Ibarz M, Martínez-Alonso M, Falguera M, Porcel JM (2008) C-reactive protein and other predictors of poor outcome in patients hospitalized with exacerbations of chronic obstructive pulmonary disease. Respirology 13:10281033. https://doi.org/10.1111/J.1440-1843.2008.01403.X

40. Leuzzi G, Galeone C, Taverna F, Suatoni P, Morelli D, Pastorino U (2017) C-reactive protein level predicts mortality in COPD: a systematic review and meta-analysis. Eur Respir Rev 26:28143876. https://doi.org/10.1183/16000617.0070-2016

41. Guilian WEI, Zhang Z (2019) Clinical value of hs-CRP and BNP detection in the diagnosis and treatment of COPD patients with pulmonary heart disease. Chin J Prim Med Pharm 12:1429-1432

42. Sato Y, Yoshihisa A, Oikawa M et al (2019) Prognostic impact of chronic obstructive pulmonary disease on adverse prognosis in hospitalized heart failure patients with preserved ejection fraction-a report from the JASPER registry. J Cardiol 73:459-465. https://doi.org/10.1016/j.jjcc.2019.01.005

Publisher's Note Springer Nature remains neutral with regard to jurisdictional claims in published maps and institutional affiliations. 